\title{
Correction to: Fatou closedness under model uncertainty
}

\author{
Marco Maggis $^{1} \cdot$ Thilo Meyer-Brandis ${ }^{2} \cdot$ Gregor Svindland $^{2}$
}

Published online: 26 November 2018

(C) Springer Nature Switzerland AG 2018

\section{Correction to: Positivity (2018) 22:1325-1343 https://doi.org/10.1007/s11117-018-0578-1}

There is an error in Proposition 3.10. In fact, the stated proof only shows

Proposition 0.1 If $c a_{c}^{*}=L_{c}^{\infty}$, then $L_{c}^{\infty}$ is order complete. Conversely, if $L_{c}^{\infty}$ is order complete and if the order continuous dual of $L_{c}^{\infty}$ may be identified with $c a_{c}$, then $c a_{c}^{*}=L_{c}^{\infty}$.

This affects Examples 3.11 and 3.12 in which the additional condition that the order continuous dual of $L_{c}^{\infty}$ may be identified with $c a_{c}$ has to be added. We thank Felix-Benedikt Liebrich for discussions which made us recognize the error.

Publisher's Note Springer Nature remains neutral with regard to jurisdictional claims in published maps and institutional affiliations.

The original article can be found online at https://doi.org/10.1007/s11117-018-0578-1.

Gregor Svindland

svindla@math.lmu.de

Marco Maggis

marco.maggis@unimi.it

Thilo Meyer-Brandis

meyerbra@math.lmu.de

1 Department of Mathematics, University of Milan, Milan, Italy

2 Mathematics Institute, LMU Munich, Munich, Germany 